\title{
A synthetic-lethality RNAi screen reveals an ERK-mTOR co- targeting pro-apoptotic switch in PIK3CA+ oral cancers
}

\author{
Kosuke Yamaguchi' ${ }^{1}$, Ramiro Iglesias-Bartolomé ${ }^{2}$, Zhiyong Wang ${ }^{1}$, Juan Luis \\ Callejas-Valera ${ }^{1}$, Panomwat Amornphimoltham ${ }^{1}$, Alfredo A. Molinolo슬 Ezra E. \\ Cohen $^{1}$, Joseph A. Califano ${ }^{1}$, Scott M. Lippman'1, Ji Luo ${ }^{3}$ and J. Silvio Gutkind ${ }^{1,4}$ \\ 1 Moores Cancer Center, University of California San Diego, San Diego, CA, USA \\ ${ }^{2}$ Laboratory of Skin Biology, National Institute of Arthritis and Musculoskeletal and Skin Diseases, National Institutes of \\ Health, Bethesda, MD, USA \\ ${ }^{3}$ Laboratory of Cancer Biology and Genetics, Center for Cancer Research, National Cancer Institute (CCR-NCI), National \\ Institutes of Health, Bethesda, MD, USA \\ ${ }^{4}$ Oral and Pharyngeal Cancer Branch, National Institute of Dental and Craniofacial Research, National Institutes of Health, \\ Bethesda, MD, USA
}

Correspondence to: J. Silvio Gutkind, email: sgutkind@ucsd.edu

Keywords: synthetic lethality screen, shRNA library, rapamycin, trametinib, co-targeting therapy

Received: January 15, $2016 \quad$ Accepted: January 29, $2016 \quad$ Published: February 13, 2016

\section{ABSTRACT}

mTOR inhibition has emerged as a promising strategy for head and neck squamous cell carcinomas (HNSCC) treatment. However, most targeted therapies ultimately develop resistance due to the activation of adaptive survival signaling mechanisms limiting the activity of targeted agents. Thus, co-targeting key adaptive mechanisms may enable more effective cancer cell killing. Here, we performed a synthetic lethality screen using shRNA libraries to identify druggable candidates for combinatorial signal inhibition. We found that the ERK pathway was the most highly represented. Combination of rapamycin with trametinib, a MEK1/2 inhibitor, demonstrated strong synergism in HNSCC-derived cells in vitro and in vivo, including HNSCC cells expressing the HRAS and PIK3CA oncogenes. Interestingly, cleaved caspase-3 was potently induced by the combination therapy in PIK $3 \mathrm{CA}^{+}$cells in vitro and tumor xenografts. Moreover, ectopic expression of PIK3CA mutations into PIK3CA- HNSCC cells sensitized them to the pro-apoptotic activity of the combination therapy. These findings indicate that co-targeting the mTOR/ERK pathways may provide a suitable precision strategy for HNSCC treatment. Moreover, PIK3CA ${ }^{+}$HNSCC are particularly prone to undergo apoptosis after mTOR and ERK inhibition, thereby providing a potential biomarker of predictive value for the selection of patients that may benefit from this combination therapy.

\section{INTRODUCTION}

Recent advances in RNAi technology have enabled synthetic lethal screenings in mammalian cells on a genome-wide scale [1]. Synthetic lethality, first described in yeast genetics, occurs when alteration of a gene results in change of the cellular phenotype only in the presence of another nonlethal genetic alteration. Recently, this approach has been applied to mammalian cells using RNAi screens [2, 3]. For example, RNAi screens in combination with active compounds were used for the identification of sensitizing targets and novel genetic interdependencies in cancer [4,5]. RNAi screens can be carried out with either siRNA-based transient transfection or shRNA-based stable gene knockdown. Vector-based shRNA libraries have several unique advantages that make them particularly attractive: they can be screened in pools and this significantly reduces the cost of the screen; they afford long-term gene knockdown and thus can reveal slow phenotypic changes in the cell; and they can be readily adapted for in vivo screens in mouse models [1].

Head and neck squamous cell carcinomas (HNSCC) are among the ten cancers most frequently diagnosed each year in the United States, affecting approximately 
42,000 new patients and resulting in approximately 8,300 deaths [6]. Mammalian target of rapamycin (mTOR) is at the center of signaling pathways that are critical for the regulation of cellular metabolism, growth, and proliferation [7]. Recent findings indicate that multiple genetic and epigenetic alterations converge on the persistent activation of $\mathrm{PI} 3 \mathrm{~K} / \mathrm{AKT} / \mathrm{mTOR}$ signaling in most HNSCC lesions [8-13]. Specifically, activating mutations in the PI3K catalytic subunit $\alpha$, encoded by the $P I K 3 C A$ gene, is the most frequent oncogenic event in HNSCC, with $18.1 \%$ of all HNSCC displaying PIK3CA mutations and $21.2 \%$ of cases displaying $P I K 3 C A$ gene copy number gain. In addition, HNSCC also have multiple genomic alterations, such as PTEN mutations (2.8\%) and gene copy number loss $(31.0 \%)$ and activating mutations in $R A S(5.9 \%)$ and $A K T(2.2 \%)$ genes that result in PI3K/ mTOR pathway activation. This cancer driver overreliance may in turn render HNSCC particularly sensitive to PI3K and mTOR inhibitors. Indeed, we and others have demonstrated this pathway dependence in a large series of genetically-defined and chemically-induced preclinical HNSCC experimental models by inhibiting mTOR with rapamycin and its analogs, which inhibit the activity of mTORC1 via binding to FKBP-12 and forming a ternary complex with mTOR [14-18].

The use of rapamycin and rapalogs have validated the concept that the PI3K/AKT/mTOR pathway can be successfully targeted in clinical cancer treatment. In this regard, our recently completed clinical trial using rapamycin in newly diagnosed and previously untreated HNSCC patients has demonstrated promising clinical activity [19] in contrast to most tumor types in which rapalogs often have modest and highly variable responses [18]. However, most targeted agents promote the activation of adaptive cellular responses that ultimately render cancer lesions resistant to their antitumor effect [20]. Thus, the combinatorial use of mTOR inhibitors with other drugs interfering with these resistance mechanisms may represent a promising strategy to improve treatment efficacy. In order to identify new potential targets for combination treatment with mTOR inhibitors, we performed a synthetic lethality screen using a pooled shRNA library with rapamycin in HNSCC cells. We identified a synthetic lethal interaction between ERK pathway inhibition and rapamycin, and validated the synergism of the co-target treatment on the growth inhibition of HNSCC cells in vitro and in vivo. Furthermore, we found that HNSCC cells harboring $P I K 3 C A$ mutations are particularly susceptible to undergo apoptosis upon mTOR and ERK inhibition, thus providing a new therapeutic option for $P I K 3 C A^{+} \mathrm{HNSCC}$ patients.

\section{RESULTS}

\section{An RNAi screens reveals synthetic lethal interactions with mTOR inhibitors}

We utilized a pooled retroviral shRNA library for the synthetic lethal screen [21]. We analyzed the change in relative abundance of each shRNA over time by sequencing of half-hairpin $(\mathrm{HH})$ barcodes derived from the shRNA population to identify those that are antiproliferative and are thus depleted from the population. We compared the lethality signature of the cells in presence of rapamycin and control to identify those shRNAs showing selective depletion in the cells in presence of rapamycin but not in control (Figure 1A). We chose the HNSCC cell line HN12 [16] for the screen, as this HNSCC cell line carries no driver mutations endogenously [9], but, as most HNSCC-derived cells, exhibits elevated mTOR activity and it is sensitive to growth inhibition in response to rapamycin [14]. Target genes were defined as hits when the difference between $\log 2 \mathrm{end} / \mathrm{start}$ ratio of rapamycin treated samples and that of control samples was $\leq-2$. We identified 117 genes as hits, among which 22 encoded kinases (Figure 1B). This gene set was particularly enriched for kinases involved in ERK pathway signaling, including KSR1 (KSR1), CRAF/ RAF1 (RAF1), ERK (MAPK1) and RSK1 (RPS6KA1), which suggests that their depletion by shRNAs is synthetically lethal with rapamycin. We also performed gene ontology (GO) enrichment analysis and found the GO term "MAPK cascade" was significantly related to this gene set $(P=2.7 \mathrm{E}-8)$. These findings prompted us to explore the impact of ERK signaling inhibition in HNSCC cells in combination with rapamycin. As an approach, we took advantage that a selective MEK1/2 inhibitor trametinib, which blocks ERK activation, was recently approved for the treatment of unresectable or metastatic melanoma with $B R A F^{\mathrm{V} 600 \mathrm{E}}$ or $B R A F^{\mathrm{V} 600 \mathrm{~K}}$ mutations by the US Food and Drug Administration as a monotherapy and in combination with dabrafenib [22].

In order to evaluate the effect of drug-drug interaction on cell viability, we performed a factorial dose matrix combinatorial drug treatment with rapamycin and trametinib. HN12 cells were incubated with these drugs in a $6 \times 8$ dose-response matrix and cell viability was measured after $72 \mathrm{hrs}$ of treatment (Figure 1C). We also investigated whether the combination effect displayed a synergistic activity. For this purpose, the fraction affected (Fa) combination index (CI) plot (Fa-CI plot) curves were simulated using CompuSyn software [23]. CI at 0.5 of Fa was 0.14 and 0.10 for $1: 4$ and $1: 16$ constant ratio of rapamycin to trametinib respectively (Figure 1D). These data indicated a strong synergism of the combination therapy in HN12 cells. We next evaluated the effect of 
A

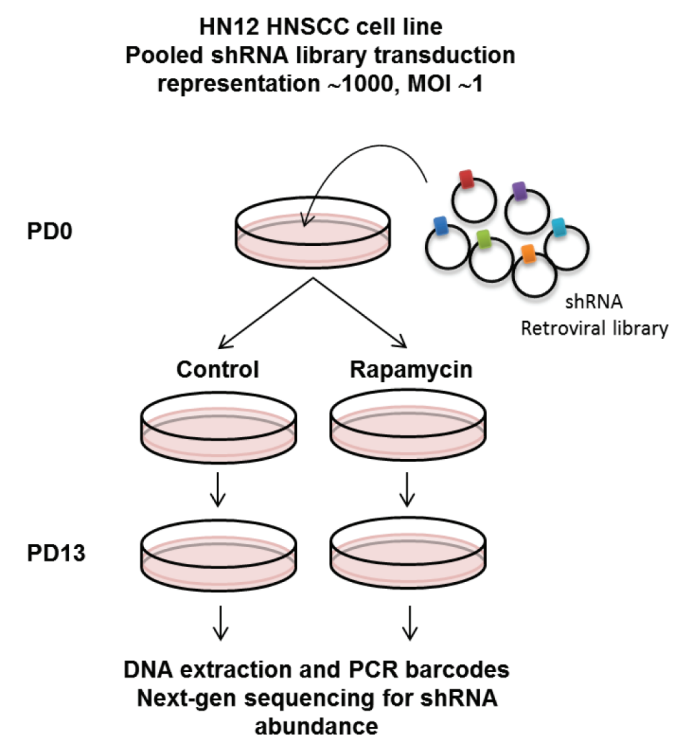

C

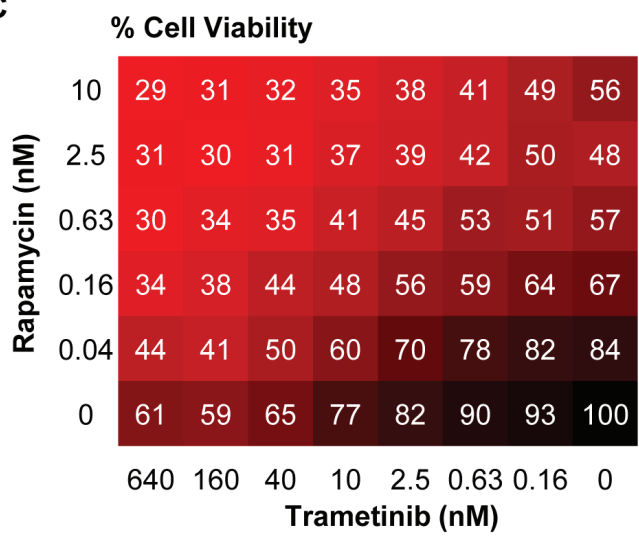

D

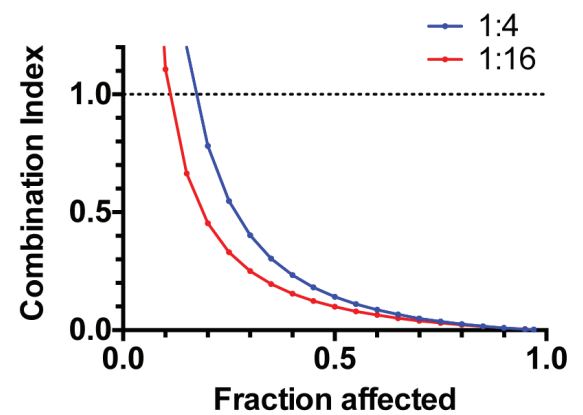

B

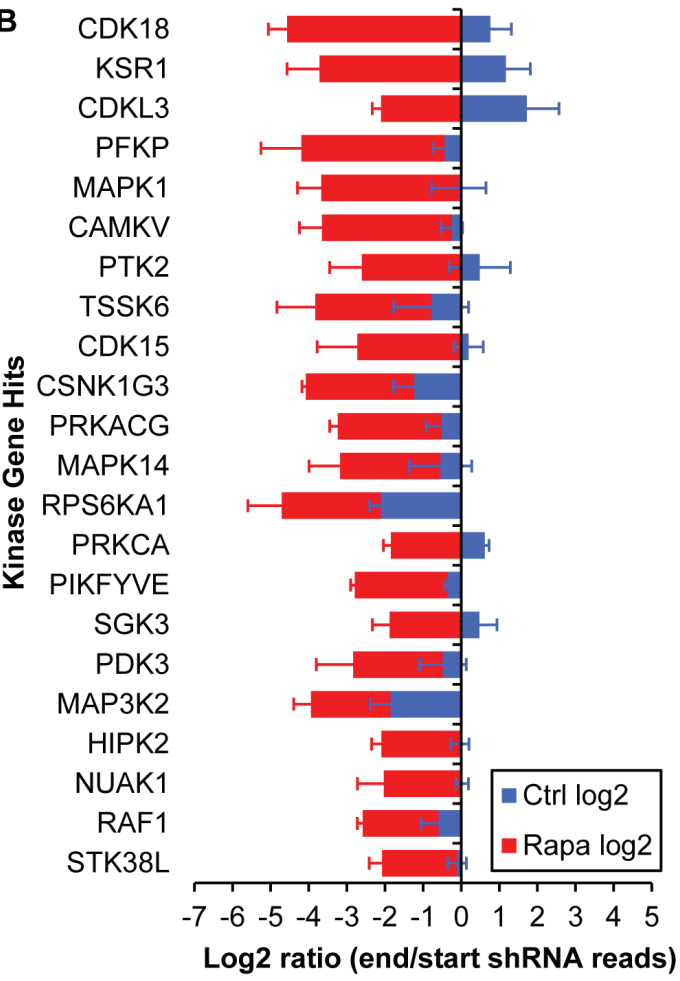

E

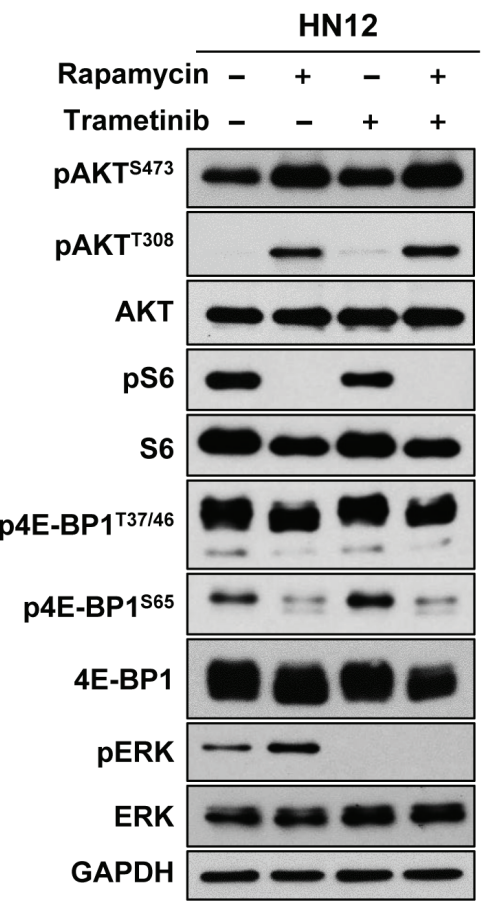

Figure 1: RNAi synthetic lethal screen. A. Schema of shRNA screen. The change in the relative abundance of each shRNA in the library over time is measured using the normalized PD 13/PD 0 ratio of its reads. A log2 PD 13/PD 0 ratio of $<0$ indicates the shRNA is depleted in the population over time, and a $\log 2$ PD 13/PD 0 ratio of $>0$ indicates the shRNA is enriched in the population. B. Hit kinase genes. Targets were filtered by $\log 2$ PD 13/PD 0 ratio of $<-2(n=3)$. C. Factorial dose matrix combinatorial drug treatment. HN12 cells were incubated for $72 \mathrm{hrs}$ with indicated concentrations of drugs. Numbers on the matrix indicate \% Cell Viability $(n=3)$. D. Computersimulated Fa-CI curves were created based on the matrix data. The ratios of rapamycin : trametinib were indicated. Synergism $(\mathrm{CI}<$ $1)$, additive effect $(\mathrm{CI}=1)$, or antagonism $(\mathrm{CI}>1)$ for the indicated levels of growth inhibition (Fraction affected) induced by the drug combination. E. mTOR/ERK pathway. HN12 cells were treated with $0.1 \%$ DMSO, 20nM rapamycin, 20nM trametinib or the combination for $24 \mathrm{hrs}$. 
rapamycin and trametinib on the ERK and PI3K-mTOR signaling pathway in these HNSCC cells. For this purpose, cells were seeded in 6-well plates and harvested after $24 \mathrm{~h}$ treatment, and samples were used for western blotting analysis. As expected, rapamycin decreased phospho-S6 (pS6) and phospho-4E-BP1 (p4E-BP1) (Figure 1E). In contrast, phospho-AKT (pAKT) and phospho-ERK (pERK) were increased (Figure 1E). Instead, trametinib clearly decreased pERK, consistent with its MEK blocking activity, and of interest, trametinib also partially decreased pS6 (Figure 1E).

A
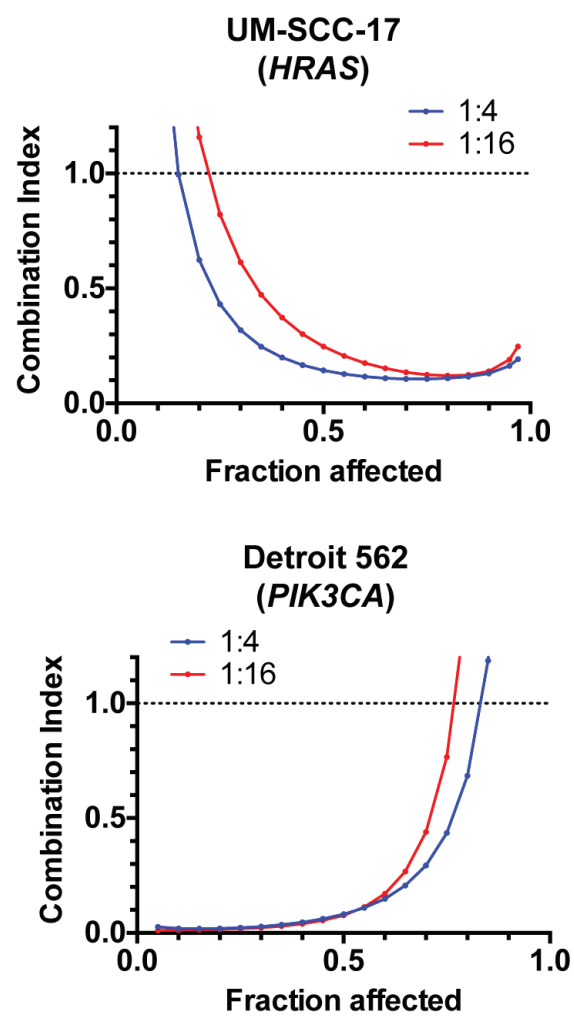

Synergistic effects of the combination of rapamycin and trametinib on HNSCC harboring HRAS and PIK3CA mutations

HNSCC lesions often harbor activating mutations in PIK3CA (18.1\%), encoding the catalytic PI3K- $\alpha$ subunit and less frequent oncogenic mutants of the $H R A S$ (5.6 $\%)$ or $K R A S(0.2 \%)$ genes [8, 12], collectively referred herein as $R A S$. To explore whether the combination of rapamycin and trametinib also displays synergistic effect in HNSCC tumors harboring activating mutations of $R A S$ and $P I K 3 C A$, we evaluated the impact of this drug

B

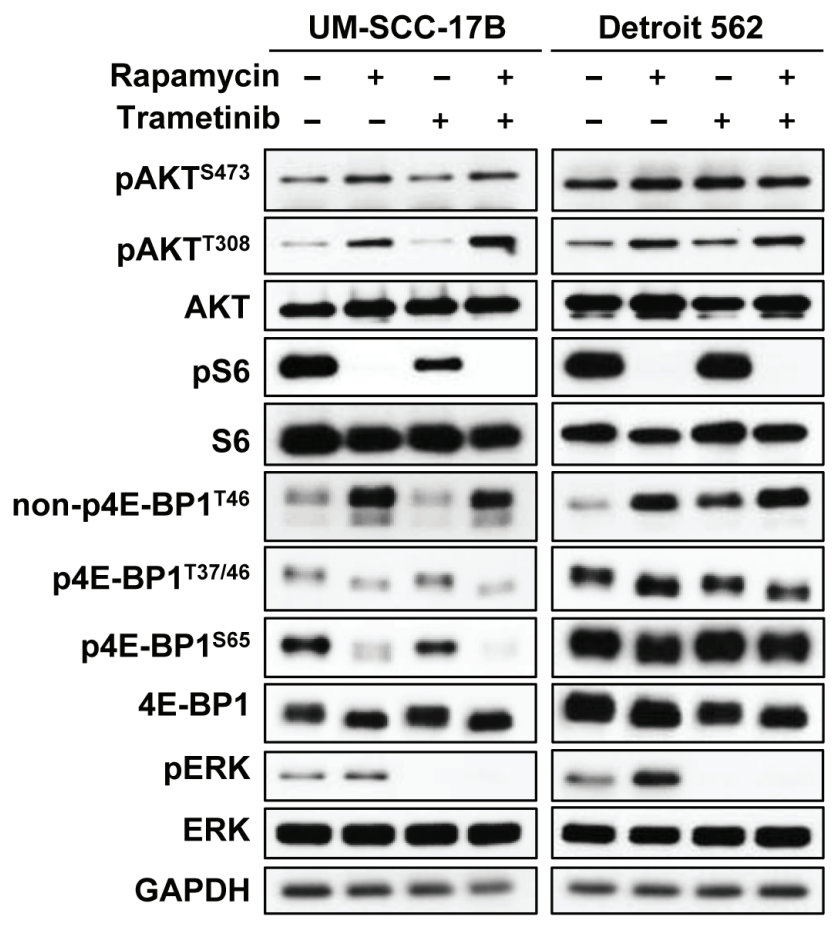

C

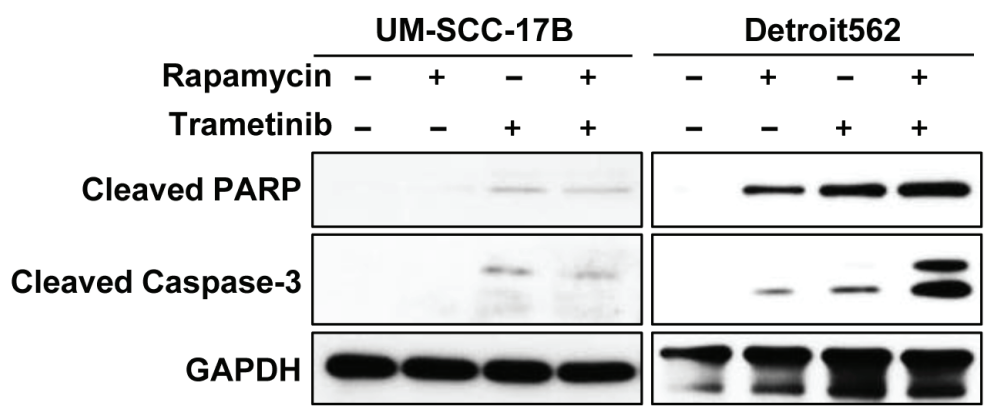

Figure 2: Synergistic effects of the combination of rapamycin and trametinib on HNSCC-derived cells harboring HRAS or PIK3CA mutations. A. Computer-simulated Fa-CI curves were created based on the factorial dose matrix combinatorial drug treatment. The ratios of rapamycin : trametinib were indicated. Synergism $(\mathrm{CI}<1)$, additive effect $(\mathrm{CI}=1)$, or antagonism $(\mathrm{CI}>1)$ for the indicated levels of growth inhibition (Fraction affected) induced by the drug combination. B. mTOR/ERK signaling pathway. Cells were treated with $0.1 \%$ DMSO, $20 \mathrm{nM}$ rapamycin, $20 \mathrm{nM}$ trametinib or the combination for $24 \mathrm{hrs}$. C. Immunoblot analysis for cleavedPARP and cleaved caspase-3. Cells were treated with $0.1 \%$ DMSO, 20nM rapamycin, 20nM trametinib or the combination for $24 \mathrm{hrs}$. 

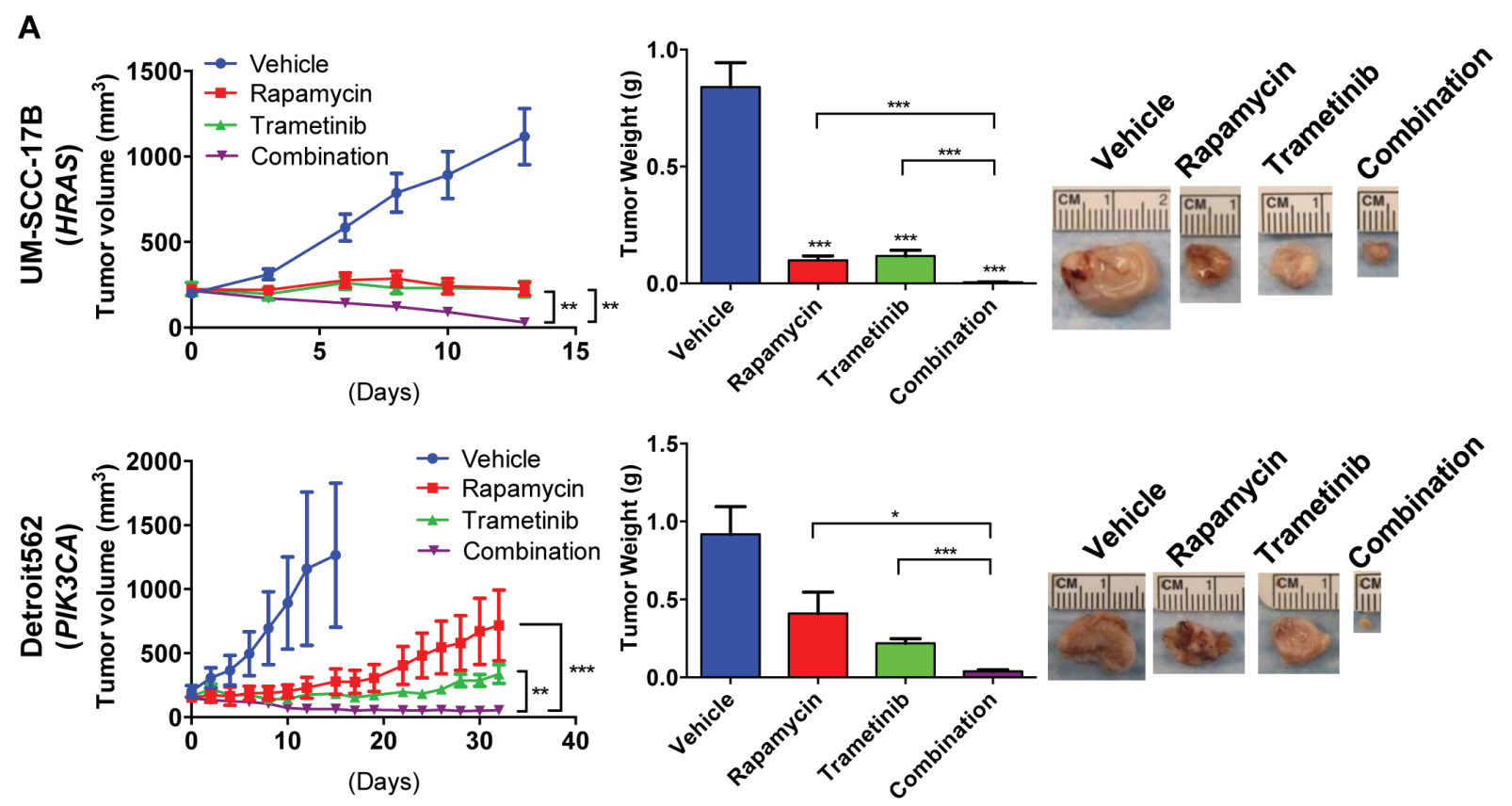

B
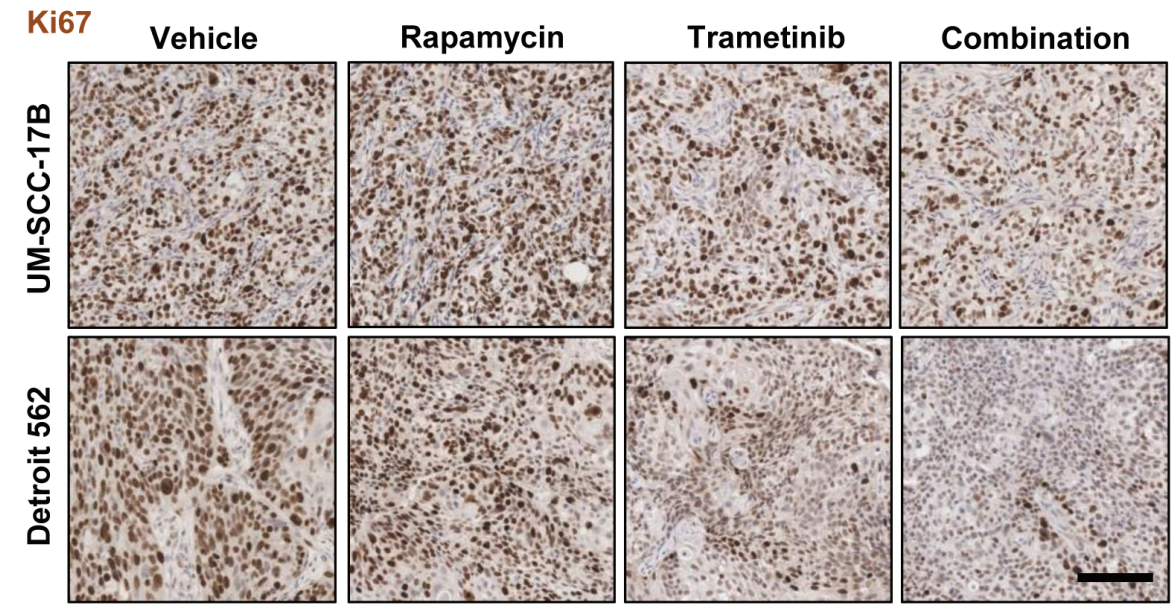

UM-SCC-17B

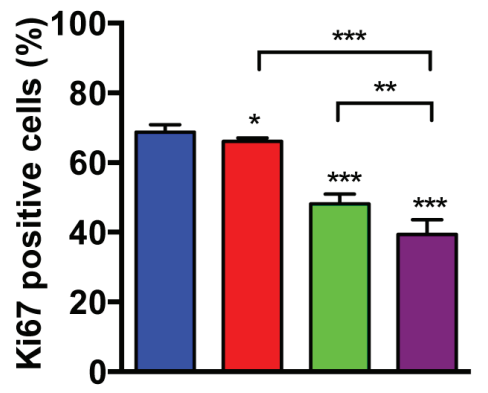

Detroit 562

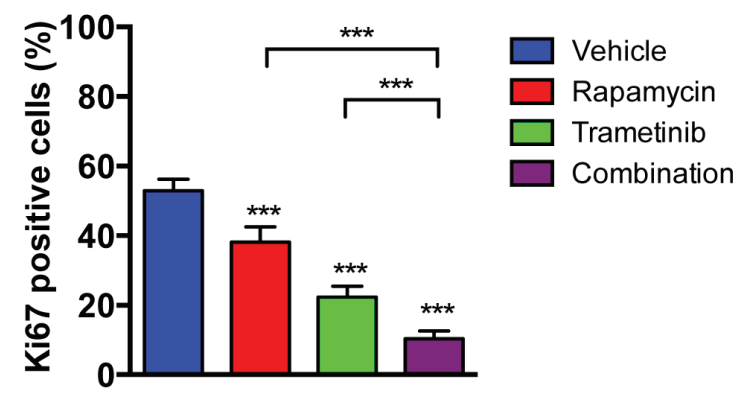

Figure 3: Antitumoral activity of the mTOR ERKcombination therapy with rapamycin and trametinib against HNSCC harboring HRAS or PIK3CA mutations. A. Antitumor efficacy of rapamycin, trametinib, and combination. Athymic nude mice were transplanted with HNSCC cells. Treatment was initiated when the tumor volume reached approximately $200 \mathrm{~mm}^{3}$. The tumor growth curves (left), tumor weights at the end of the single agent and combined treatments (middle) and pictures of representative tumors (right) are displayed. Data points represent mean values $\pm \operatorname{SE}(n=10$ per each group). B. Representative tumor tissue sections (top) and quantification (bottom) stained for Ki67 ( $n=6$ per each group). Scale bars represent $100 \mu \mathrm{m} .{ }^{*} P<0.05,{ }^{* *} P<0.01,{ }^{* * *} P<0.001$. 
combination in HNSCC cells harboring endogenous oncogenic mutations. We used UM-SCC-17B, which has a HRAS Q61L mutant and Detroit 562 cells exhibiting the PIK3CA H1047R mutation. The combination of rapamycin and trametinib demonstrated strong synergism in growth inhibition of these cells (Figure 2A, Supplementary Figure S1A). We next evaluated the effect of the combination treatment in the downstream signaling pathway of RAS and PI3K in these cell lines. Rapamycin decreased pS6 and p4E-BP1, but in contrast, pAKT and pERK were both increased (Figure 2B). Trametinib treatment clearly decreased pERK, and it also enhanced the rapamycinmediated decrease of pS6 and p4E-BP1 (Figure 2B). We also evaluated whether trametinib, rapamycin, or their combination can promote apoptosis as judged by the accumulation of cleaved caspase-3. Remarkably, we found that whereas these drugs alone have limited effect, the combination treatment induced a significant increase in apoptosis in PIK3CA mutant HNSCC cell line, Detroit 562 , but not in the HRAS mutant HNSCC cells UM-SCC17B (Figure 2C).

\section{Antitumoral activity of the combination therapy with rapamycin and trametinib against HNSCC harboring HRAS or PIK3CA mutations}

We next evaluated the antitumoral activity of the ERK-mTOR targeting combination therapy in vivo, using UM-SCC-17B and Detroit 562 HNSCC xenografts, which harbor endogenous activating $H R A S$ and $P I K 3 C A$ mutations, respectively. The combination therapy with rapamycin and trametinib was more effective than each of the single agents individually (Figure 3A). The body weight after 4 weeks treatment was $22.7 \pm 2.1 \mathrm{~g}$ in the combination treatment group and $23.7 \pm 0.7 \mathrm{~g}$ in vehicle control group. The antitumoral activity was reflected by a decreased immunohistochemical detection of nuclear Ki67, which was used for the evaluation of cell proliferation. The MEK-mTOR combination therapy showed significant reduction of HNSCC cells expressing nuclear Ki67 in both xenograft models (Figure 3B). Immunohistochemistry analysis of treated tumors revealed that trametinib enhanced the rapamycin-mediated inhibition of S6 and 4E-BP1 in vivo, and blunted the rapamycin-induced ERK activation in these HNSCC cells (Figure 4A, 4B). Consistent with the activation of ERK, rapamycin also induced elevated phospho-MEK1/2 (pMEK 1/2) levels. pMEK1/2 was increased by trametinib, likely as a feedback mechanisms stimulating the activation of MEK upstream kinases upon MEK inhibition by its direct blocker. AKT was activated by rapamycin in UMSCC-17B but not in Detroit 562 in vivo, suggesting that activation of the ERK pathway may represent a more general secondary effect of rapamycin than pAKT increase (Figure 4A). In addition, a clear distinction between these
HNSCC cells was that cleaved caspase- 3 was significantly increased by the combination therapy in Detroit 562 (PIK3CA mutant) but not in UM-SCC-17B (HRAS mutant) (Figure 4C).

\section{mTOR and MEK inhibition display a synergistic growth inhibitory activity in HNSCC cells genetically engineered to express activating $K R A S$ and $P I K 3 C A$ mutations}

Our observations suggested that mTOR and MEK co-targeting exerts a synergistic antiproliferative effect in HNSCC cells in vitro and in vivo, and a selective proapoptotic impact on PIK3CA expressing HNSCC cells. However, cancer cells display an intrinsic heterogeneity, hence it is unclear if trametinib and rapamycin promote apoptosis in Detroit 562 because these cells express mutant $P I K 3 C A$ or if this response is due to cell-dependent variations. As an approach to explore these possibilities, we next investigated whether a significant synergism was displayed in isogenic HNSCC cells, CAL27 cells, which were genetically engineered to express $R A S$ and $P I K 3 C A$ mutants [17]. Strong synergism was demonstrated between trametinib and rapamycin in wild type CAL27 cells and in both KRAS and PIK3CA expressing CAL27 cells, supporting that the drug combination is effective in HNSCC cells displaying multiple genetic alterations (Figure 5A, Supplementary Figure S1B). In this biological relevant system, $P I 3 K C A$ expressing CAL27 cells showed significantly stronger synergism than CAL27 WT and CAL27 KRAS cells. Indeed, CI at ED $\mathrm{ED}_{50}$ was $0.09,0.06$ and 0.01 for CAL27 WT, CAL27 KRAS and CAL27 PIK3CA cells, respectively (Figure 5A).

We next evaluated the effect of rapamycin and trametinib in the downstream signaling pathway elicited by RAS and PI3K in these cells. As for other HNSCC cells, rapamycin decreased pS6 and p4E-BP1 in these cell lines. In contrast, pAKT and pERK were increased (Figure 5B). Trametinib clearly decreased pERK and enhanced the rapamycin-mediated reduction of pS6 and p4E-BP1 (Figure 5B). We also evaluated apoptosis by cleaved caspase-3 (Figure 5C). Rapamycin increased caspase-3 cleavage in CAL27 PIK3CA cells but failed to stimulate apoptosis in the CAL27 WT cell line. Trametinib also promoted a limited increase in cleaved caspase- 3 in CAL27 KRAS, and this effect was much more remarkable in CAL27 PIK3CA cells (Figure 5C). 
A
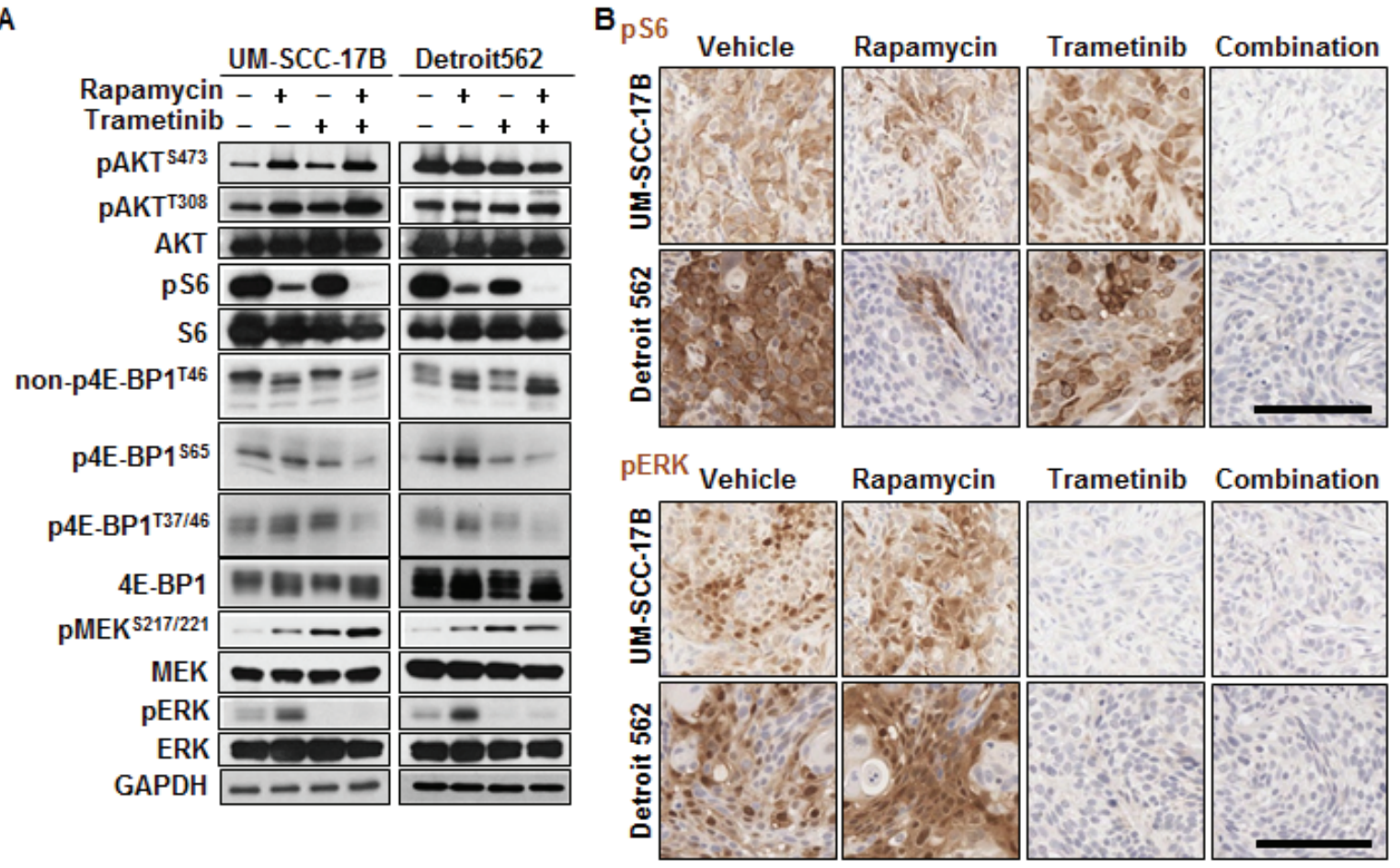

C Cleaved Caspase-3
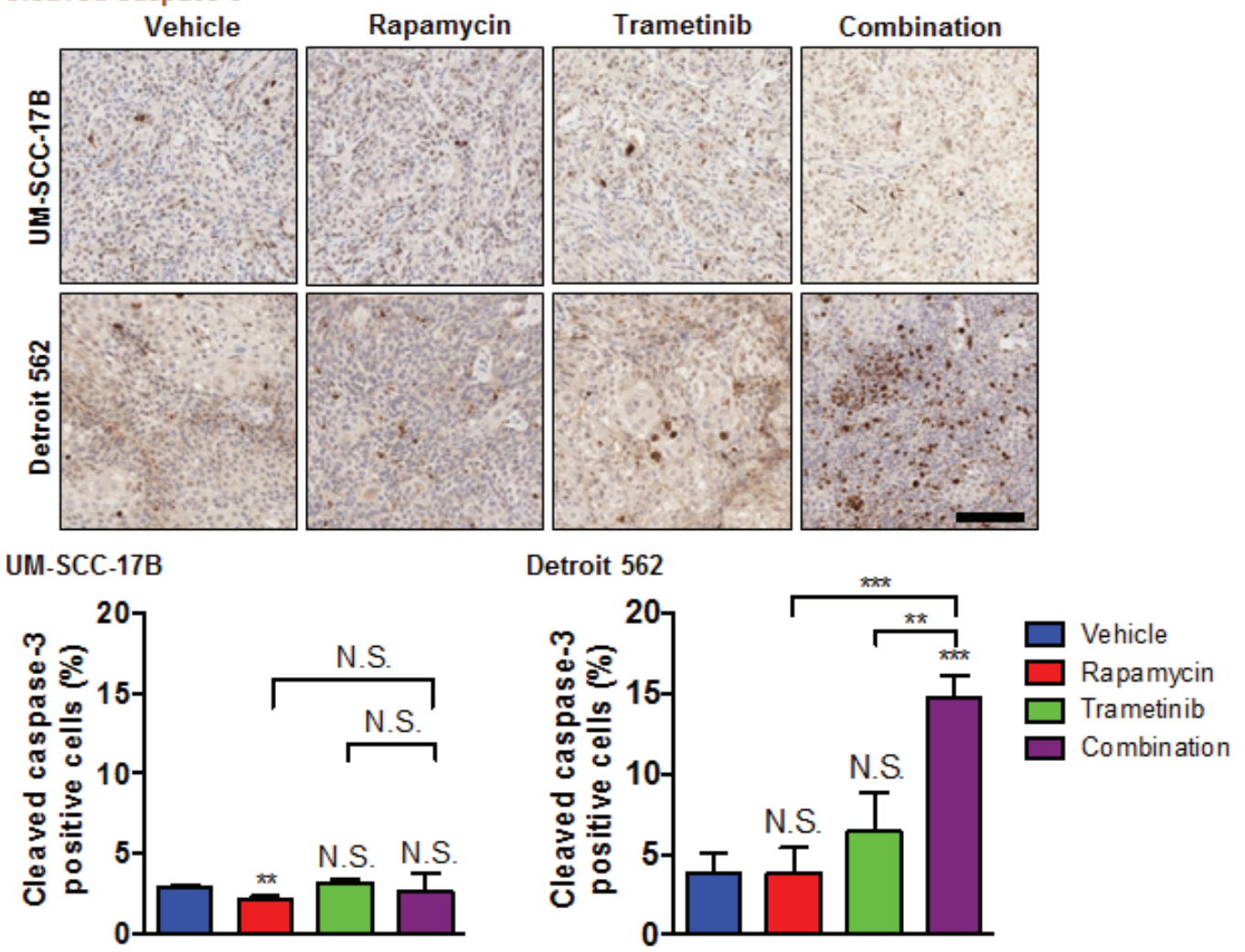

Figure 4: Effects of the combination of rapamycin and trametinib on mTOR/ERK signaling and apoptosis in HNSCC harboring HRAS or PIK3CA mutations. A. mTOR/ERK signaling pathway. Cells were transplanted into athymic mice and treated with vehicle, rapamycin, trametinib or combination for four days. Tumor lysates were analyzed with immunoblot analysis. B. Representative tumor tissue sections stained for pS6 and pERK. Scale bars represent $100 \mu \mathrm{m}$. C. Representative tumor tissue sections (top) and quantification (bottom) stained for cleaved-caspase 3 ( $n=3$ per each group). Scale bars represent $100 \mu \mathrm{m} . * P<0.05, * * P<0.01,{ }^{* * *} P$ $<0.001$. 
Antitumoral activity of the rapamycin and trametinib combination therapy in genetically engineered HNSCC cells expressing activating $R A S$ or PIK $3 C A$ mutations

We next investigated whether the combination of rapamycin and trametinib was effective in the isogenic cell line panel CAL27 WT, CAL27 HRAS and CAL27 PIK3CA xenograft models. A more significant antitumoral effect was observed in the groups treated with the combination therapy than in the groups treated with single agents in each of these xenograft models (Figure 6A). This was reflected by decreased nuclear Ki67, a biomarker for cell proliferation, in the combination therapy compared to single agents (Figure 6B, Supplementary Figure S2A). To study the biochemical and biological effects of the drug combination in vivo in this isogenic HNSCC cell panel, we performed immunohistochemistry for $\mathrm{pS} 6$, pERK (Figure 7A) and cleaved caspase-3 (Figure 7B). Rapamycin mediated inhibition of pS6 was enhanced by the combination with trametinib (Figure 7A). ERK was activated by rapamycin in these cells, and strongly inhibited by the therapy with trametinib (Figure 7A).
A
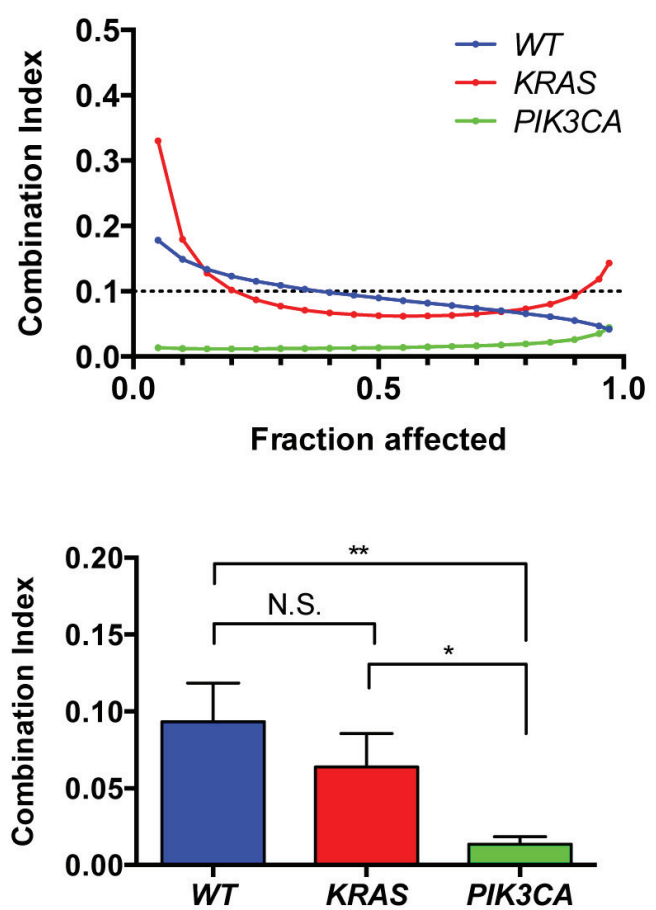

B
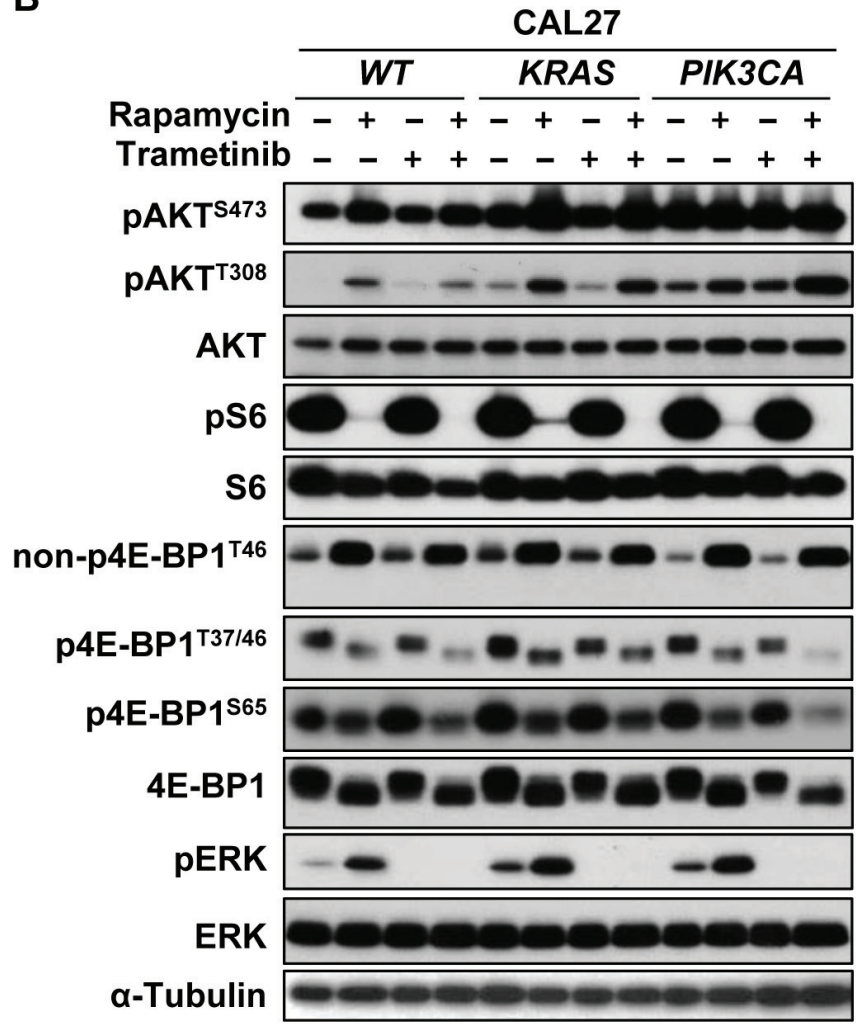

C

\begin{tabular}{|c|c|c|c|c|c|c|c|c|c|c|c|}
\hline \multirow{3}{*}{$\begin{array}{l}\text { Rapamycir } \\
\text { Trametinit }\end{array}$} & \multicolumn{4}{|c|}{ WT } & \multicolumn{4}{|c|}{ KRAS } & \multicolumn{3}{|c|}{ PIK 3 CA } \\
\hline & - & + & - & + & - & + & - & + & - & $+\quad-$ & + \\
\hline & - & - & + & + & - & - & + & + & - & + & + \\
\hline Cleaved Caspase-3 & & & - & . & & & - & - & & -2 & \pm \\
\hline GAPDH & & & & & & & & & & & \\
\hline
\end{tabular}

Figure 5: Synergism of rapamycin and trametinib in genetically engineered HNSCC cells to express activating KRAS or PIK3CA mutations. A. Computer-simulated Fa-CI curves were created based on the factorial dose matrix combinatorial drug treatment. The ratios of rapamycin : trametinib were 1:16. Synergism $(\mathrm{CI}<1)$, additive effect $(\mathrm{CI}=1)$, or antagonism $(\mathrm{CI}>1)$ for the indicated levels of growth inhibition (Fraction affected) induced by the drug combination (left). CI values at $\mathrm{Fa}=0.5$ was used to calculate the mean between experiments $(n=3)$. ${ }^{*} P<0.05,{ }^{*} P<0.01$. B. mTOR/ERK signaling pathway. Cells were treated with $0.1 \%$ DMSO, $20 \mathrm{nM}$ rapamycin, $20 \mathrm{nM}$ trametinib or the combination for $0.5 \mathrm{~h}$ or $24 \mathrm{hrs}$. C. Immunoblot analysis for cleaved-caspase 3 . Cells were treated with $0.1 \%$ DMSO, $20 \mathrm{nM}$ rapamycin, $20 \mathrm{nM}$ trametinib or the combination for $24 \mathrm{hrs}$. 
A

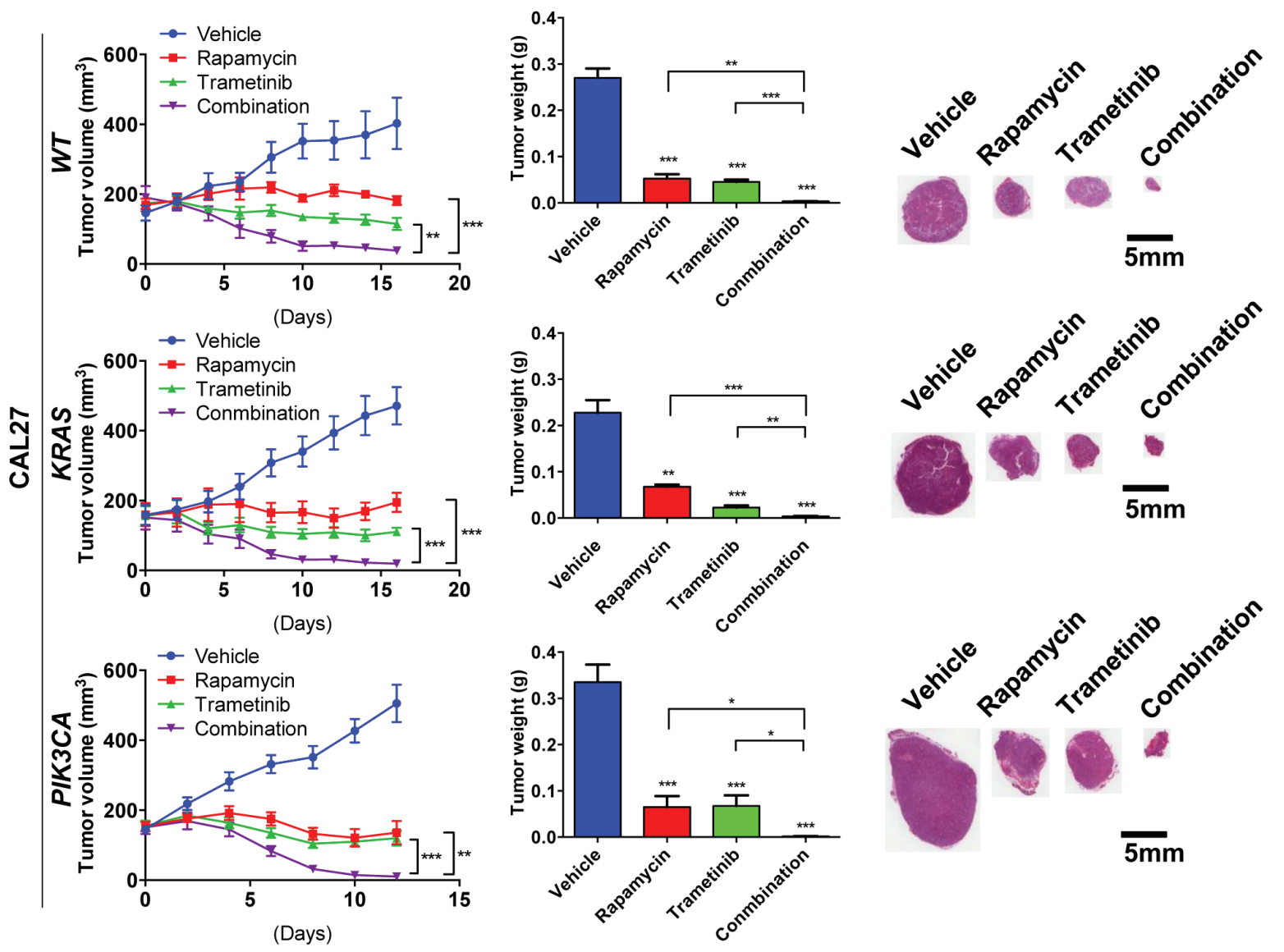

B $\quad$ Ki67

CAL27 PIK3CA

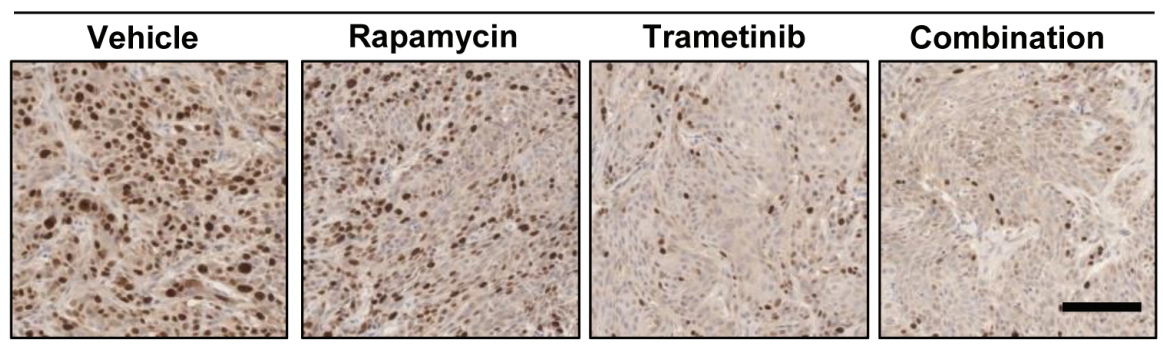

$W T$

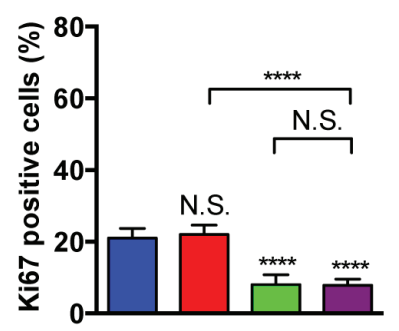

KRAS

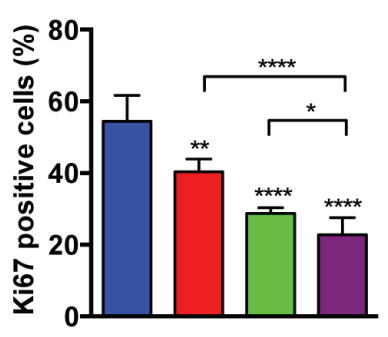

PIK3CA

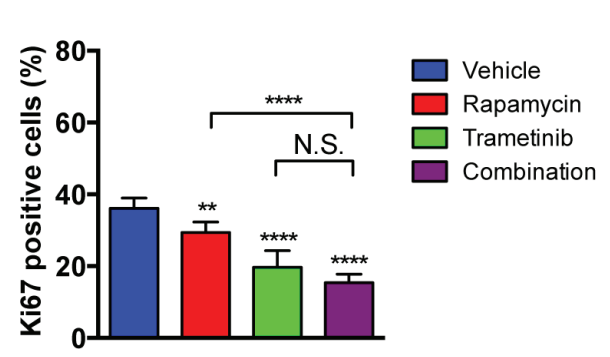

Figure 6: Antitumoral activity of the rapamycin and trametinib combination therapy against genetically engineered HNSCC cells expressing KRAS and PIK3CA oncogenes. A. Antitumor efficacy of rapamycin, trametinib, and combination. Athymic nude mice were transplanted with HNSCC cells. Treatment was initiated when the tumor volume reached approximately 200 $\mathrm{mm}^{3}$. The tumor growth curves (left), tumor weights at the end of the single agent and combined treatments (middle) and representative histological sections for each treatment group. Scale bars represent $5 \mathrm{~mm}$ (right) are displayed. Data points represent mean values $\pm \mathrm{SE}(n=$ 10 per each group). B. Representative tumor tissue sections (top) and quantification (bottom) stained for Ki67 ( $n=6$ per each group). Scale bars represent $100 \mu \mathrm{m} .{ }^{*} P<0.05, * * P<0.01, * * * P<0.001$. 
Cleaved caspase-3 was significantly induced in the tumors treated with the combination therapy in CAL27 PIK3CA (Figure 7B). However, only minor pro-apoptotic effects were observed in CAL27 WT and CAL27 KRAS (Figure 7B, Supplementary Figure S2B). These finding suggest that while co-targeting MEK and $\mathrm{mTOR}$ is effective for tumor reduction in all $\mathrm{HNSCC}$ models tested, PIK3CA expression specifically sensitizes HNSCC cells to the proapoptotic activity of MEK and mTOR combined targeted treatment, thereby displaying increased response.

\section{DISCUSSION}

Our in vitro data demonstrated strong synergism of the combination treatment with rapamycin and trametinib against HNSCC -derived cells exhibiting quite distinct genomic alterations, including cells carrying endogenous and genetically engineered $R A S$ or $P I K 3 C A$ mutations. Furthermore, the co-targeting therapy demonstrated significant effect on tumor growth in all HNSCC xenograft models tested. Intriguingly, CAL27 engineered to express PIK3CA mutations displayed the strongest synergism in vitro. Furthermore, the combination treatment significantly induced cleaved-caspase 3 in HNSCC cell lines carrying $P I K 3 C A$ mutation endogenously and when ectopically expressed. Thus, in the presence of PIK3CA mutations, adding MEK inhibitors to mTOR blockade causes a proapoptotic switch from cytostatic to cytotoxic, which has important beneficial therapeutic implications.

The mechanism of this remarkable genotypespecific drug interaction remains unclear. For example, the ERK pathway has profound effects on the regulation
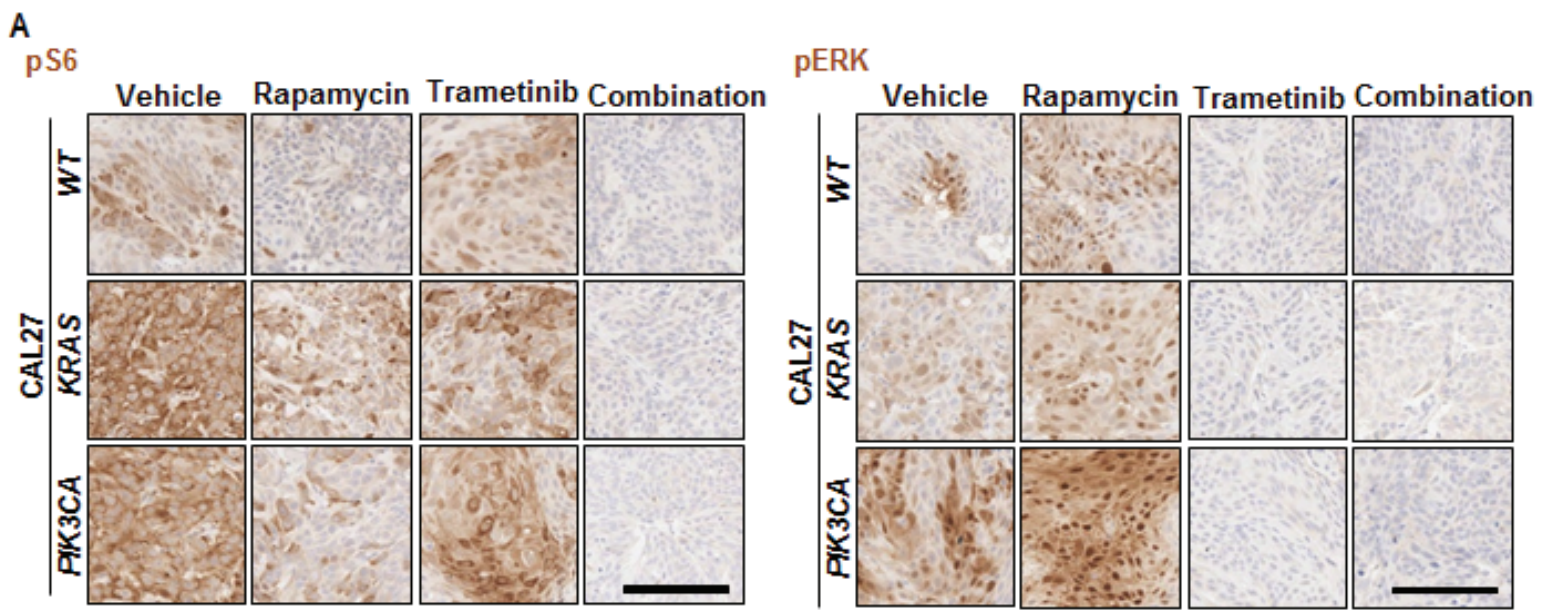

B

Cleaved Caspase-3

CAL27 PIK3CA
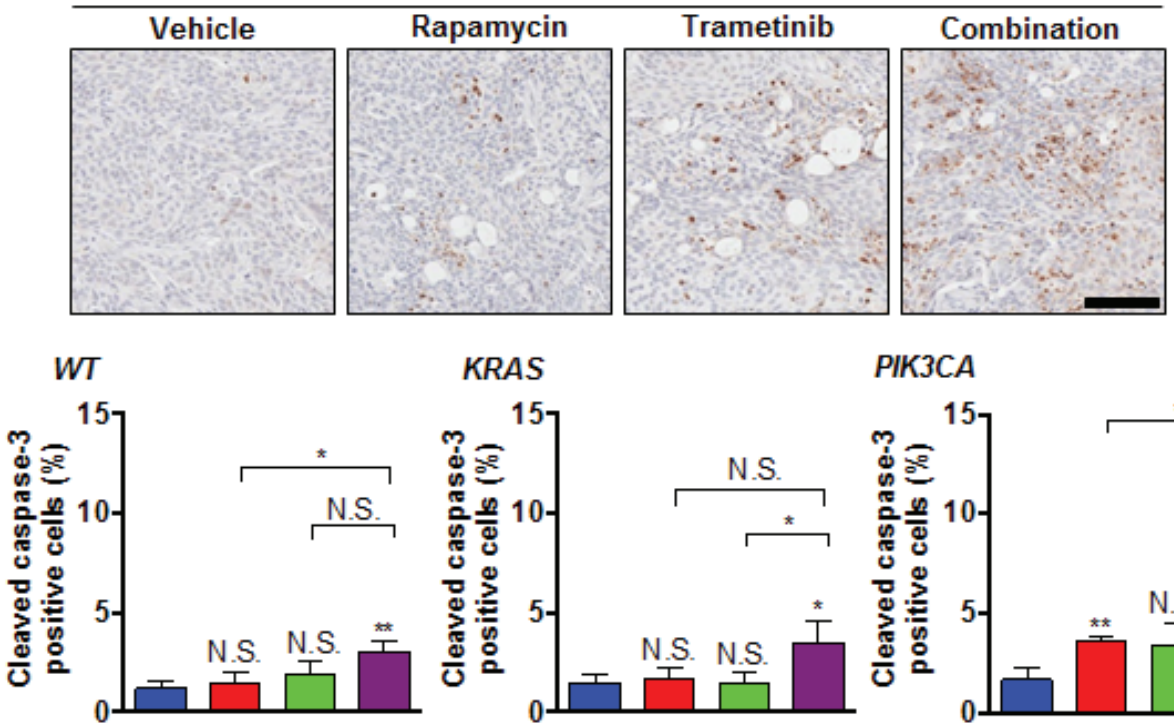

\section{KRAS}

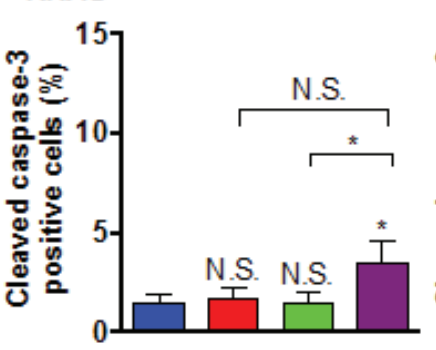

\section{PIKЗCA}

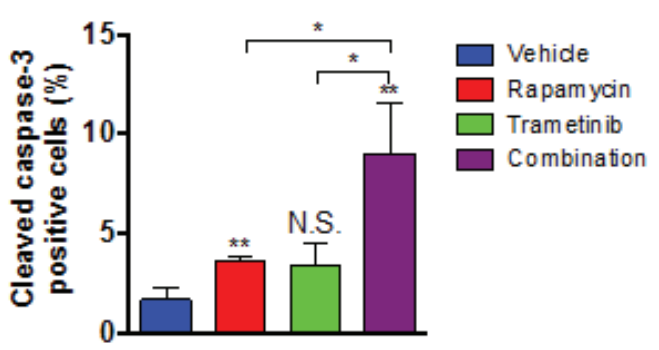

Figure 7: Effects of the combination of rapamycin and trametinib on mTOR/ERK signaling and apoptosis in genetically engineered HNSCC cells KRAS and PIK3CA mutations. A. Representative tumor tissue sections stained for pS6 and pERK. Scale bars represent $100 \mu \mathrm{m}$. B. Representative tumor tissue sections (top) and quantification (bottom) stained for cleaved-caspase 3 ( $n=3$ per each group). Scale bars represent $100 \mu \mathrm{m} . * P<0.05, * * P<0.01$. 
of cell survival pathways by the post-translational phosphorylation of pro-apoptotic and apoptotic regulatory molecules, including BAD, BIM, Mcl-1, caspase 9 and Bcl-2 [24]. On the other hand, mTOR also possesses both pro-apoptotic and anti-apoptotic effects [25]. Activated S6K is capable of binding to mitochondrial membrane and phosphorylating the pro-apoptotic molecule BAD, rendering $\mathrm{BAD}$ inactive and promoting cell survival [26]. In line with these observations, mTOR inhibition can promote apoptosis in several cancer models [16, 27]. Conversely, several studies demonstrated that mTOR inhibition attenuates cytotoxic agent-induced apoptosis in malignant cells [28, 29]. The mTOR function on apoptosis appears to be dictated by cell type and its activation state as well as by the mutational status of its downstream targets including well known apoptosisregulatory proteins such p53, BAD and Bcl-2 [30]. Thus, the interplay between mTOR and ERK inhibition may have pro-apoptotic functions in restricted genetic contexts. In line with this possibility, recently, Hata et al. reported that the concomitant use of MEK and PI3K inhibitor lead to upregulation of PUMA and BIM, both of which were necessary for the induction of an apoptotic response in $K R A S$-mutant in another cancer type, nonsmall cell lung cancer (NSCLC) $[31,32]$. Certainly, the underlying reasons in $\mathrm{HNSCC}$ that an apoptotic response is only elicited in PIK3CA mutant cells is clearly clinically relevant, and its precise underlying mechanism warrants further investigation.

In the current study we identified 22 kinases whose suppression is synthetic lethal with rapamycin for therapy against HNSCC. These include KSR1 (KSR1), CRAF/ RAF1 (RAF1), ERK (MAPK1) and RSK1 (RPS6KA1), which are involved in the ERK signaling pathway, suggesting that interfering with ERK activation renders HNSCC cells more sensitive to mTOR inhibition. Indeed, we show here that co-targeting MEK and mTOR using FDA approved therapeutic agents, trametinib and rapamycin, respectively, exerts a synergistic antiproliferative and pro-apoptotic effect in all HNSCC cell lines tested irrespective of their quite distinct mutational genomic landscape [9]. Furthermore, we found that the combined drug therapy was much more effective in promoting tumor regression that any of the single agents alone, supporting that synthetic gene lethality screens can be exploited for the development of new therapeutic options for HNSCC treatment.

In addition to the ERK pathway, we also identified p38 MAPK (MAPK14) and MAP3K2 (MAP3K2), which are involved in other MAPK pathways as cotargeting candidates. Recently, we reported that p38 MAPK functions as a positive regulator of HNSCC in the context of the tumor microenvironment, controlling cancer cell growth and tumor-induced angiogenesis and lymphangiogenesis [33]. We also identified kinases involved in PI3K/AKT pathway. Among these were SGK3
(SGK3), ARK5/NUAK1 (NUAK1), and NDR2 (STK38L). For example, activation of SGK3 downstream of PIK3CA and INPP4B is required for 3D cell proliferation, invasive migration, and tumorigenesis in vivo [34]. Overexpression of ARK5 confers tolerance to glucose starvation, which is a stress that leads to a decrease in ATP and an increase in AMP [35]. Activated AKT stimulates cell invasion by phosphorylating ARK5 at Ser600 [35]. ARK5 has been also reported to act as a tumor invasion-associated factor through NDR2 during IGF-1 signaling [36]. We also identified FAK (PTK2) and HIPK2 (HIPK2) in our synthetic lethality screens. FAK promotes cell survival through kinase-dependent and kinase-independent mechanisms $[37,38]$. Overall, these results suggest that several MAPK and PI3K/AKT pathway components and FAK, among others, may participate in adaptive response mechanisms promoting resistance to rapamycin in HNSCC cells. Hence, their knock down or pharmacological inhibition with small molecule inhibitors can be also considered for the future development of more effective combination therapies for HNSCC.

Overall, our unbiased genetic screen revealed that co-targeting mTOR and ERK using FDA approved agents results in a remarkable synergistic interaction in HNSCC preclinical models. While the inhibition of the PI3K/ $\mathrm{AKT} / \mathrm{mTOR}$ and ERK pathways has been investigated in other malignancies, the in vivo impact of this cotargeting strategy for HNSCC has not been well validated. Indeed, in all HNSCC tumors tested, the combination of tramatinib and rapamycin caused a rapid tumor collapse, and was much more active than any of these agents alone. Furthermore, we now show that the concomitant inhibition of mTOR and MEK promotes the apoptotic demise of HNSCC cells specifically in tumors expressing the $P I K 3 C A$ oncogene, the most frequently driver mutation in this cancer type $[8,12]$. Thus, these findings will have significant clinical implications, as they define a new mechanism-based precision therapeutic approach for the treatment of HNSCC patients, and define PIK $3 C A$ as a suitable biomarker of heightened beneficial therapeutic response to the combined treatment.

\section{MATERIALS AND METHODS}

\section{Cell lines, tissue culture and antibodies}

Human head and neck cancer cell lines Cal27, Detroit 562, were from American Type Culture Collection; UM-SCC-17B cell line was from Thomas Carey, University of Michigan. Cells were cultured in Dulbecco's modified Eagle's medium with $10 \%$ fetal bovine serum supplemented with antibiotics, $5 \% \mathrm{CO}_{2}$ at $37{ }^{\circ} \mathrm{C}$. All cell lines underwent DNA authentication by multiplex STR profiling (Genetica DNA Laboratories, 
Inc. Burlington, NC) prior to the described experiments to ensure consistency in cell identity. Antibodies against AKT, pAKT ${ }^{\mathrm{S} 473}, \mathrm{pAKT}^{\mathrm{T} 308}$, ERK1/2, pERK1/2, S6, pS6, MEK1/2, pMEK1/2, 4E-BP1, non-p4E-BP1 ${ }^{\text {T46 }}$, p4E$\mathrm{BP} 1^{\mathrm{T} 37 / 46}$, p4E-BP1 ${ }^{\mathrm{s} 65}$, cleaved caspase-3, cleaved PARP, $\alpha$-Tubulin-HRP, GAPDH were purchased from Cell Signaling Technology (Beverly, MA). Mouse anti-Ki67 antibody was purchased from DAKO (Carpinteria, CA).

\section{shRNA screening}

shRNA screening was performed as described [21]. HN12 cells were infected with pools of retroviral shRNA at a representation of $\sim 1,000$ and a multiplicity of infection (MOI) of $\sim 1$. At day 3 post infection cells were selected with puromycin for an additional 3 days $(1 \mu \mathrm{g} /$ $\mathrm{ml})$ to remove the minority of uninfected cells. After that, cells where propagated in culture for 3 days and then an initial population-doubling 0 (PD 0) sample was taken. The rest of the population was divided in 6 groups, and 3 groups where kept as a control and 3 where treated with rapamycin $(100 \mathrm{nM})$. Cells where propagated in the presence or not of drug for an additional 12 doublings before the final, PD 13 sample was taken. For each passage a minimal representation of 1000 was maintained. The shRNA library contained 9,149 retroviral shRNAs targeting human kinases, phosphatases, genes involved in protein ubiquination, and genes implicated in cancer. The libraries where expressed using the retroviral vector MSCV-PM. For PD 0 and PD 13 samples, shRNA HH barcode was PCR-recovered from genomic samples and samples where sequenced to calculate abundance of the different shRNA probes.

The change in the relative abundance of each shRNA in the library over time is measured using the normalized PD 13/PD 0 ratio of its reads. A log2 PD 13/PD 0 ratio of $<0$ indicates the shRNA is depleted in the population over time, and a $\log 2$ PD 13/PD 0 ratio of $>0$ indicates the shRNA is enriched in the population. To identify shRNAs that are synthetically lethal with rapamycin, the mean log2 PD 13/PD 0 ratios of the rapamycin treated cell triplicates were compared to that of the control triplicates to derive the $\log 2$ ratio difference. A p-value of the difference between the two triplicates was calculated using the $t$-test. Targets were filtered by the presence of at least 2 different shRNAs for the same gene and a $P<0.05$. GO enrichment analysis was performed with ToppGene Suite software[39].

\section{Cell growth assays and drug combination analysis}

Alamar Blue Cell Viability Reagent was purchased from Life Technologies. (Grand Island, NY) Cells were cultured in 96-well-plate and treated with drugs for 72 hours. The manufacturer's instructions were followed to complete the assay. Drug synergy was determined by the combination index methods using CompuSyn software [23]. CI values less than 1 , equal to 1 , and greater than 1 represent synergism, additivity, and antagonism, respectively.

\section{Immunoblot analysis}

The cells and the tissues were lysed in RIPA buffer containing Halt protease and phosphatase inhibitor cocktail (Thermo Scientific, Rockford, IL). Protein concentration was measured by Bio-Rad Protein Assay (Bio-Rad, Hercules, CA). Equal amounts of total proteins were subjected to SDS-polyacrylamide gel electrophoresis and transferred to PVDF membranes. Membranes were blocked with $5 \%$ nonfat dry milk in T-TBS buffer $(50 \mathrm{mM}$ Tris/HCl, pH 7.5, 0.15 M NaCl, 0.1\% [v/v] Tween-20) for $2 \mathrm{~h}$, and then incubated with primary antibodies in blocking buffer at $4{ }^{\circ} \mathrm{C}$ overnight. Detection was conducted by incubating the membranes with horseradish peroxidase-conjugated goat anti-rabbit IgG secondary antibody (Southern Biotech, Birmingham, AL) was used at a dilution of 1:50,000 in 5\% milk-T-TBS buffer, at room temperature for $1 \mathrm{~h}$, and visualized with Immobilon Western Chemiluminescent HRP Substrate (Millipore, Billerica, MA).

\section{Tissue analysis}

All samples were fixed in zinc formalin (Z-Fix, Anatech) and embedded in paraffin; $5 \mu \mathrm{m}$ sections were stained with Hematoxylin-Eosin for diagnostic purposes. For immunohistochemistry (IHC) studies the sections were deparaffinized, hydrated through graded ethanols, and the endogenous peroxidase inhibited with $3 \% \mathrm{H}_{2} \mathrm{O}_{2}$ in $70 \%$ ethanol. The slides were extensively washed with distilled water and antigen retrieval was performed by high temperature treatment with $10 \mathrm{mM}$ citric acid in a microwave. After washing with water and PBS, the slides were successively incubated with the primary and secondary antibodies, and the $\mathrm{ABC}$ reagent (Vector Laboratories, Burlingame, CA). The reaction was developed with 3-3'-diamonobenzifdine under microscopic control. A Mouse on Mouse (M.O.M.) Basic Kit (Vector Laboratories, Burlingame, CA) was used in Ki67 staining to inhibit binding of secondary antibodies to mouse tissue.

\section{In vivo mouse experiments and analysis}

All the mice studies were carried out according to National Institutes of Health (NIH) approved protocols (ASP \#13-695) in compliance with the NIH Guide for the Care and Use of Laboratory Mice. To establish tumor 
xenografts, cells were transplanted into the flanks of athymic nude mice (female, four to six weeks old) (Harlan Laboratories, Inc., Indianapolis, IN), and when the tumor volume reached approximately $200 \mathrm{~mm}^{3}$, the mice were randomized into groups and treated by intraperitoneal injection (ip) with trametinib ( $1 \mathrm{mg} / \mathrm{kg} /$ day) and rapamycin ( $5 \mathrm{mg} / \mathrm{kg} /$ day), or control diluent ( $n=10$ per each group). Tumor volume was calculated by using the formula length $\times$ width $\times$ width $/ 2$. The mice were euthanized at the indicated time points and tumors isolated for histologic and immunohistochemical evaluation.

\section{Data and statistical analysis}

Data were analyzed using GraphPad Prism version 6 (GraphPad Software, San Diego, CA). Comparisons between experimental groups were made using unpaired $t$ test. $P<0.05$ was considered to be statistically significant.

\section{CONFLICTS OF INTEREST}

The authors declare no conflict of interest.

\section{REFERENCES}

1. $\mathrm{Hu} \mathrm{G}$ and Luo J. A primer on using pooled shRNA libraries for functional genomic screens. Acta biochimica et biophysica Sinica. 2012; 44:103-112.

2. Simons A, Dafni N, Dotan I, Oron Y and Canaani D. Establishment of a chemical synthetic lethality screen in cultured human cells. Genome research. 2001; 11:266-273.

3. Simons AH, Dafni N, Dotan I, Oron Y and Canaani D. Genetic synthetic lethality screen at the single gene level in cultured human cells. Nucleic acids research. 2001; 29:E100.

4. Bernards R, Brummelkamp TR and Beijersbergen RL. shRNA libraries and their use in cancer genetics. Nature methods. 2006; 3:701-706.

5. Seyhan AA, Varadarajan U, Choe S, Liu Y, McGraw J, Woods M, Murray S, Eckert A, Liu W and Ryan TE. A genome-wide RNAi screen identifies novel targets of neratinib sensitivity leading to neratinib and paclitaxel combination drug treatments. Molecular bioSystems. 2011; 7:1974-1989.

6. Siegel R, Ma J, Zou Z and Jemal A. Cancer statistics, 2014. CA Cancer J Clin. 2014; 64:9-29.

7. Ma XM and Blenis J. Molecular mechanisms of mTORmediated translational control. Nature reviews Molecular cell biology. 2009; 10:307-318.

8. Iglesias-Bartolome R, Martin D and Gutkind JS. Exploiting the head and neck cancer oncogenome: widespread PI3KmTOR pathway alterations and novel molecular targets. Cancer discovery. 2013; 3:722-725.

9. Martin D, Abba MC, Molinolo AA, Vitale-Cross L,
Wang Z, Zaida M, Delic NC, Samuels Y, Lyons JG and Gutkind JS. The head and neck cancer cell oncogenome: a platform for the development of precision molecular therapies. Oncotarget. 2014; 5:8906-8923. doi: 10.18632/ oncotarget. 2417.

10. Molinolo AA, Marsh C, El Dinali M, Gangane N, Jennison K, Hewitt S, Patel V, Seiwert TY and Gutkind JS. mTOR as a molecular target in HPV-associated oral and cervical squamous carcinomas. Clinical cancer research. 2012; 18:2558-2568.

11. Molinolo AA, Amornphimoltham P, Squarize CH, Castilho RM, Patel V and Gutkind JS. Dysregulated molecular networks in head and neck carcinogenesis. Oral oncology. 2009; 45:324-334.

12. Cancer Genome Atlas N. Comprehensive genomic characterization of head and neck squamous cell carcinomas. Nature. 2015; 517:576-582.

13. Hedberg ML, Goh G, Chiosea SI, Bauman JE, Freilino ML, Zeng Y, Wang L, Diergaarde BB, Gooding WE, Lui VW, Herbst RS, Lifton RP and Grandis JR. Genetic landscape of metastatic and recurrent head and neck squamous cell carcinoma. The Journal of clinical investigation. 2015.

14. Amornphimoltham P, Patel V, Sodhi A, Nikitakis NG, Sauk JJ, Sausville EA, Molinolo AA and Gutkind JS. Mammalian target of rapamycin, a molecular target in squamous cell carcinomas of the head and neck. Cancer research. 2005; 65:9953-9961.

15. Amornphimoltham $\mathrm{P}$, Leelahavanichkul K, Molinolo A, Patel V and Gutkind JS. Inhibition of Mammalian target of rapamycin by rapamycin causes the regression of carcinogen-induced skin tumor lesions. Clinical cancer research. 2008; 14:8094-8101.

16. Amornphimoltham P, Patel V, Leelahavanichkul K, Abraham RT and Gutkind JS. A retroinhibition approach reveals a tumor cell-autonomous response to rapamycin in head and neck cancer. Cancer research. 2008; 68:11441153.

17. Wang Z, Martin D, Molinolo AA, Patel V, IglesiasBartolome R, Degese MS, Vitale-Cross L, Chen Q and Gutkind JS. mTOR co-targeting in cetuximab resistance in head and neck cancers harboring PIK3CA and RAS mutations. Journal of the National Cancer Institute. 2014; 106.

18. Meng LH and Zheng XF. Toward rapamycin analog (rapalog)-based precision cancer therapy. Acta pharmacologica Sinica. 2015; 36:1163-9. doi: 10.1038/ aps.2015.68.

19. Shirai K, Day TA, Szabo E, Van Waes C, O’Brien PE, Matheus MG, Godwin K, Sood AJ, Vick JA, Martin D, Vitale-Cross L, Molinolo A and Gutkind JS. A pilot, single arm, prospective trial using neoadjuvant rapamycin prior to definitive therapy in head and neck squamous cell carcinoma. Journal of Clinical Oncology. 2015; 33.

20. Rebecca VW and Smalley KS. Change or die: targeting 
adaptive signaling to kinase inhibition in cancer cells. Biochemical pharmacology. 2014; 91:417-425.

21. Luo J, Emanuele MJ, Li D, Creighton CJ, Schlabach MR, Westbrook TF, Wong KK and Elledge SJ. A genome-wide RNAi screen identifies multiple synthetic lethal interactions with the Ras oncogene. Cell. 2009; 137:835-848.

22. Mandal R, Becker S and Strebhardt K. Stamping out RAF and MEK1/2 to inhibit the ERK1/2 pathway: an emerging threat to anticancer therapy. Oncogene. 2015.

23. Chou TC. Theoretical basis, experimental design, and computerized simulation of synergism and antagonism in drug combination studies. Pharmacological reviews. 2006; 58:621-681.

24. McCubrey JA, Steelman LS, Chappell WH, Abrams SL, Wong EW, Chang F, Lehmann B, Terrian DM, Milella M, Tafuri A, Stivala F, Libra M, Basecke J, et al. Roles of the Raf/MEK/ERK pathway in cell growth, malignant transformation and drug resistance. Biochimica et biophysica acta. 2007; 1773:1263-1284.

25. Nguyen SA, Walker D, Gillespie MB, Gutkind JS and Day TA. mTOR inhibitors and its role in the treatment of head and neck squamous cell carcinoma. Current treatment options in oncology. 2012; 13:71-81.

26. Harada H, Andersen JS, Mann M, Terada N and Korsmeyer SJ. p70S6 kinase signals cell survival as well as growth, inactivating the pro-apoptotic molecule BAD. Proceedings of the National Academy of Sciences of the United States of America. 2001; 98:9666-9670.

27. Stephan S, Datta K, Wang E, Li J, Brekken RA, Parangi $\mathrm{S}$, Thorpe PE and Mukhopadhyay D. Effect of rapamycin alone and in combination with antiangiogenesis therapy in an orthotopic model of human pancreatic cancer. Clinical cancer research. 2004; 10:6993-7000.

28. Calastretti A, Bevilacqua A, Ceriani C, Vigano S, Zancai P, Capaccioli S and Nicolin A. Damaged microtubules can inactivate BCL-2 by means of the mTOR kinase. Oncogene. 2001; 20:6172-6180.

29. Markova B, Hahnel PS, Kasper S, Herbertz S, Schuler M and Breitenbuecher F. Pharmacologic inhibition of mTOR antagonizes the cytotoxic activity of pemetrexed in nonsmall cell lung cancer. Journal of cancer research and clinical oncology. 2012; 138:545-554.

30. Castedo M, Ferri KF and Kroemer G. Mammalian target of rapamycin (mTOR): pro- and anti-apoptotic. Cell death and differentiation. 2002; 9:99-100.

31. Hata AN, Yeo A, Faber AC, Lifshits E, Chen Z, Cheng KA, Walton Z, Sarosiek KA, Letai A, Heist RS, Mino-Kenudson M, Wong KK and Engelman JA. Failure to induce apoptosis via BCL-2 family proteins underlies lack of efficacy of combined MEK and PI3K inhibitors for KRAS-mutant lung cancers. Cancer research. 2014; 74:3146-3156.

32. Hata AN, Engelman JA and Faber AC. The BCL2 Family: Key Mediators of the Apoptotic Response to Targeted Anticancer Therapeutics. Cancer discovery. 2015; 5:475-
487.

33. Leelahavanichkul K, Amornphimoltham P, Molinolo AA, Basile JR, Koontongkaew S and Gutkind JS. A role for p38 MAPK in head and neck cancer cell growth and tumorinduced angiogenesis and lymphangiogenesis. Molecular oncology. 2014; 8:105-118.

34. Gasser JA, Inuzuka H, Lau AW, Wei W, Beroukhim R and Toker A. SGK3 mediates INPP4B-dependent PI3K signaling in breast cancer. Molecular cell. 2014; 56:595607.

35. Sun X, Gao L, Chien HY, Li WC and Zhao J. The regulation and function of the NUAK family. Journal of molecular endocrinology. 2013; 51:R15-22.

36. Suzuki A, Ogura T and Esumi H. NDR2 acts as the upstream kinase of ARK5 during insulin-like growth factor-1 signaling. The Journal of biological chemistry. 2006; 281:13915-13921.

37. Sulzmaier FJ, Jean C and Schlaepfer DD. FAK in cancer: mechanistic findings and clinical applications. Nature reviews Cancer. 2014; 14:598-610.

38. Pylayeva Y, Gillen KM, Gerald W, Beggs HE, Reichardt LF and Giancotti FG. Ras- and PI3K-dependent breast tumorigenesis in mice and humans requires focal adhesion kinase signaling. The Journal of clinical investigation. 2009; 119:252-266.

39. Chen J, Bardes EE, Aronow BJ and Jegga AG. ToppGene Suite for gene list enrichment analysis and candidate gene prioritization. Nucleic acids research. 2009; 37:W305-311. 